\title{
The spur planetary gear torsional stiffness and its crack sensitivity under quasi-static conditions
}

\author{
Song Xue, Rodney Entwistle, Ilyas Mazhar and Ian Howard* \\ Department of Mechanical Engineering, Curtin University, Bentley, Western Australia, Australia \\ Department of Mechanical Engineering, Curtin University, GPO Box U1987, Perth, WA 6845, \\ Australia \\ * Corresponding author. Phone: (+61) 892667047. \\ E-mail: i.howard@curtin.edu.au
}

Abstract: The sun-planet and ring-planet tooth mesh stiffness variations and the resulting transmission errors are the main internal vibration generation mechanisms for planetary gear systems. This paper presents the results of torsional stiffness analysis of involute spur planetary gear systems in mesh using finite element methods. A planetary gear model with three planet gears and fixed ring gear and its subsystem models have been developed to study the subsystem and overall torsional stiffnesses. Based on the analysis of torsional mesh stiffness, predictive models for single branch sun-planet-ring and overall planetary gear torsional stiffnesses have been proposed. A crack coefficient was introduced to the sun-planet and ring-planet meshes to predict the effect and sensitivity of changes to the overall torsional mesh stiffness. The resulting mesh stiffness crack sensitivity of the overall gear system was analysed under quasi-static conditions. It was found that the carrier arm stiffness has great influence on the crack sensitivity while the overall stiffness was most sensitive to the crack on the sun-planet mesh.

Keyword: planetary gear, FEA method, subsystem torsional stiffness, overall torsional stiffness, crack sensitivity 


\section{Introduction}

Planetary gears have substantial advantages over parallel shaft drives, including compactness and large torque-to-weight ratio and because of these characteristics, planetary gear sets are widely used in many applications such as automotive, aerospace and wind turbine transmissions. However, noise and vibration, reliability assessment and early detection of damage to internal components remain major concerns in their applications. Teeth damage, including cracks, can occur due to various factors, such as improper design, manufacturing irregularities, inadequate lubrication and transient operational loads. Once a crack occurs, the subsystem mesh stiffness will change and consequently cause changes in overall stiffness and dynamic system response.

Most research on planetary gear dynamics has employed lumped-parameter models to analysis the dynamic response of the planetary gear system. Each of the sun, ring, carrier and $\mathrm{N}$ planets are treated as rigid bodies and the gear mesh interactions are represented by springs and damping acting along the line of action [1, 2]. The variations in tooth mesh stiffness are the main internal vibration generation mechanism in the system and shaft phase-variant mesh stiffness considerations are the most appropriate model for the internal excitation [1-4]. Some researches employed rectangular functions to approximate the mesh stiffness variation [3]. This approximation is useful and efficient for examining certain critical factors (like modal properties, planet load sharing) in the early design stage [2-4]. When it comes to using gear dynamic modelling to help understand and improve gearbox condition monitoring techniques, the incorporation of more realistic gear mesh stiffness functions improves the accuracy of modelling the response from a damaged gear train and can be used to generate more efficient diagnostic tools.

Analytical methods (AM) are simple and effective for evaluating the tooth mesh stiffness and in previously published papers, it has been widely used to calculate the mesh stiffness in both fixed axis gear systems and planetary gear systems [5-8]. Zaigang simplified the gear teeth using a beam model to study the effect of gear tooth crack propagation, along the tooth width for different crack depths, on the gear mesh stiffness [5, 6]. The effect of local crack and spalling failure on mesh stiffness and dynamic response has also been investigated analytically [7]. Due to non-uniform load and non-homogeneity of material, spatial cracks can occur in the gear tooth and this crack effect on mesh stiffness was studied by Wennian [8]. These studies indicate that the shape of the damaged gear tooth mesh stiffness changed compared with that from the healthy 
gear. With the effect of shaft misalignment and friction, the total gear mesh stiffness decreased compared with the healthy gear case even though there was no fault involved [9].

However, some difficult issues have been found when using the analytical method for calculating the gear mesh stiffness with the damaged tooth fault. Owing to the gear body flexibility, the extended tooth contact can occur outside the theoretical contact line [10]. The use of FE modelling was found to be most suitable for capturing the extended tooth contact phenomenon, especially when there was a crack at the gear tooth root, which can aggravate this effect. Another issue was that the deviation between the gear mesh stiffness obtained from the analytical method and the FEA method was found to become larger with the increase in the crack size. The FE model was found to be more suitable for modelling the gear tooth mesh stiffness with larger gear tooth crack size [11, 12]. In fact, the FE method has been a reliable and effective tool to study the gear mesh stiffness with or without gear tooth fault. With the principle of linear elastic fracture mechanics, the crack propagation path for gear pairs with different contact ratio was investigated [13, 14]. Shuting used 3-D FEM to study a pair of spur gears with machining errors, assembly errors and tooth modifications [15]. Vijaya used 2-D FEA to calculate the sun-planet and ring-planet mesh stiffness for a planetary gear system [16]. Jiande compared a large number of 2-D and 3-D gear models using parameters such as the torsional stiffness, tooth stresses and the stress intensity factors [17] and it was found that the results from the plane stress assumption were close to those from 3-D gear models when the gear tooth width was less than $100 \mathrm{~mm}$. With the plane stress assumption, Jiande studied the torsional stiffness of a spur gear and the effect of the tip modification on the torsional stiffness $[18,19]$.

Based on the reviews of the previous literatures, the main objective of this paper is the analysis of the torsional mesh stiffness of planetary gear systems. The development of methods to obtain the overall stiffness using FEA methods and the development of predictive models, which can give a quick estimate of the overall stiffness based on the subsystem stiffness, will be discussed. As gear cracks can deteriorate the gear system robustness and has always been a concern for gearbox condition monitoring, the predictive models developed in this research have been extended with crack coefficients to study the crack sensitivity of different components in the planetary gears. 


\section{Planetary gear FEA torsional stiffness models and analysis}

\subsection{Planetary gear FEA models}

A single stage planetary gear set usually consists of a sun gear, ring gear, several planets and a carrier. Any of the carrier, ring, and sun can be selected as the input or output component and power is transmitted through the multiple paths of the planets in mesh. In this research, the sun gear was selected as the input component and the carrier was selected as the output. The planetary gear tooth parameters used in the analysis are shown in Table 1.

Table 1 Planetary gear parameters

\begin{tabular}{llll}
\hline & Sun gear & Planet gear & Ring gear \\
\hline Number of teeth, $Z_{i}$ & $Z_{s}=21$ & $Z_{p}=39$ & $Z_{\mathrm{r}}=99$ \\
Module, $m_{n}$ & $10 \mathrm{~mm}$ & $10 \mathrm{~mm}$ & $10 \mathrm{~mm}$ \\
Pressure angle, $\alpha_{i}$ & $\alpha_{s}=20^{\circ}$ & $\alpha_{\mathrm{p}}=20^{\circ}$ & $\alpha_{\mathrm{r}}=20^{\circ}$ \\
Pitch diameter, $d_{i}=m_{n} Z_{i}$ & $d_{s}=210 \mathrm{~mm}$ & $d_{p}=390 \mathrm{~mm}$ & $d_{r}=990 \mathrm{~mm}$ \\
Elastic Modulus, $E$ & $210 \mathrm{MPa}$ & $210 \mathrm{MPa}$ & $210 \mathrm{MPa}$ \\
Poisson's Ratio, $v$ & 0.3 & 0.3 & 0.3 \\
Friction coefficient & 0.2 & 0.2 & 0.2 \\
\hline
\end{tabular}

In this gear set, it was found that the ratios $Z_{s} / \mathrm{N}=21 / 3=7$ and $Z_{r} / \mathrm{N}=99 / 3=33(\mathrm{~N}=3$, is the number of planet gears) are both integers such that all the sun-planet and ring-planet gear meshes are in phase [20]. In other situations, the mesh phasing $\gamma_{s n}$ between the various sun-planet meshes and the mesh phasing $\gamma_{r n}$ between various ring-planet meshes can be calculated according to reference [21]. However, it was found that $\gamma_{s n}=\gamma_{r n}$, which means the phase of the sun-planet and the phase of the ring-planet meshes on the same branches of the planetary gear are always identical at the same branch [21].

Finite element (FE) modelling of gears with flexible contact provide more realistic simulations and to obtain quality converged solutions, adaptive meshing should be used so the elements near the contact(s) will have relatively small dimensions that enable the analysis to cope with the chaotic non-linear nature of the contact region. No tip-relief was used in this research, apart from a round-off with a $0.1 \mathrm{~mm}$ radius at the tooth tip, to keep the original form of the involute curve. The tooth tip round off was used to assist the FE solution 
convergence where corner contact occurred. In this research, four different FEA models, as shown in Fig. 1, have been developed to study the characteristics of the planetary gear system torsional stiffness:
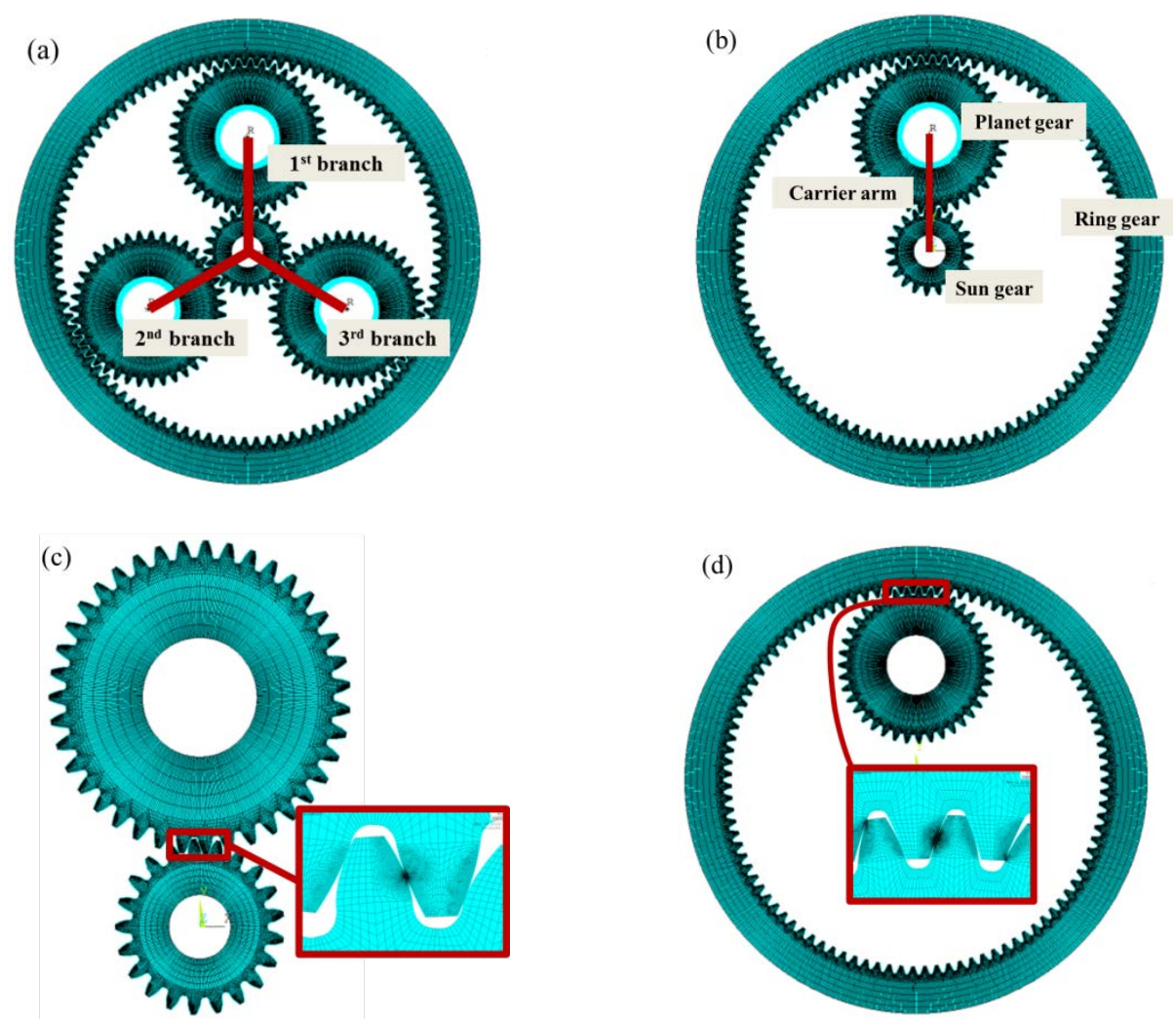

Fig. 1 FEA planetary gear models, (a). Planetary gear model with 3 planet gears and a flexible carrier arm, (b). One isolated sun-planet-ring pair with flexible carrier arm, (c).

One isolated sun-planet external pair, (d). One isolated ring-planet internal pair

The kinematic relationship between sun, planet and carrier arm can be determined by deriving the transmission ratio relationships between them. An APDL program has been developed to control the gears to be rotated to the corresponding positions before the model was solved. The starting mesh point was calculated at the mesh position of the first sun-planet gear pitch point (defined as $0^{\circ}$ ) in the first branch of the sunplanet-ring and then the sun gear was rotated clockwise with an angle increment for the next solution. In this research, with the fixed ring gear, one tooth mesh period covers the carrier rotation angle of $360^{\circ} / Z_{r}=3.64^{\circ}$ and calculations were carried out covering two mesh periods.

To avoid rigid body motion errors preventing FE convergence, a weak spring was attached to the input component hub, which was only used for the initial step with a very small torque until the teeth were just in contact. When the actual load torque was applied, the spring was disabled using the birth/death of elements command [19]. The major assumptions in this research are the following: 
(1) Plane stress assumptions were used in the 2-D FEA planetary gear model;

(2) Quasi-static conditions exist and no inertia effects influence the mesh stiffness curve;

(3) The manufacturing and assembly errors are not included and the transmission error is only due to the elastic deformation;

(4) The stiffness of the bearings is not included;

(5) The sun gear can move only in the torsional direction.

\section{$2.2 \quad$ FEA torsional stiffness results}

The gear torsional stiffness curve is a nonlinear stiffness, varying with the load and the rotation [18]. To be able to calculate the stiffness using FEA, the curve has been discretised and solved for each meshing angle and applied torque. The torsional stiffness at each point can be regarded as a linear stiffness defined as the ratio between the torque acting on the input pinion and the resulting relative elastic angular rotation between the input pinion and the output gear hubs [19],

$$
K=\frac{T}{\Delta \theta}
$$

where $T$ is the input torque load and $\Delta \theta$ is the relative elastic angular rotation between the two gears. At each particular meshing position, the angular rotation of the loaded drive gear is calculated in the gear reference frame by restraining the output gear from rotating. In ANSYS, a pilot node can be created in the centre of the hub and all the DOF (degrees of freedom) of the nodes on the edge of the hub can be coupled with the pilot node. Contact methods can be used to generate a rigid area in the hub area. After applying the input torque to the pilot node in the $M z$ direction, the value of $R O T Z$ in the FEA model will be the value of $\Delta \theta$.

\subsubsection{Isolated sun-planet (sp1) subsystem torsional stiffness}

The tooth mesh stiffness variation of a sun-planet mesh was modelled from the isolated sun-planet FE model, shown in Fig. 1(c). The sun gear hub was constrained to be fixed in the radial direction while the planet hub was fully constrained in both tangential and radial directions. However, with the sun gear rotation, the planet gear will still move around the sun gear, acting as though there were a rigid carrier arm carrying the planet gear. This will give the same movement of the planet in the overall planetary gear model. However, when the planet moves to each simulated position, its inner hub was again constrained not to rotate and the torque $T_{s p 1}$ was applied to the sun gear. If $\Delta \theta_{s p 1}$ denotes the elastic angular rotation of the sun gear hub, the torsional 
stiffness $K_{s p 1}$ can be obtained using equation (1). Different torque magnitudes were applied to the sun gear hub and the corresponding tooth mesh stiffness variation is shown in Fig. 2, covering two teeth mesh periods.

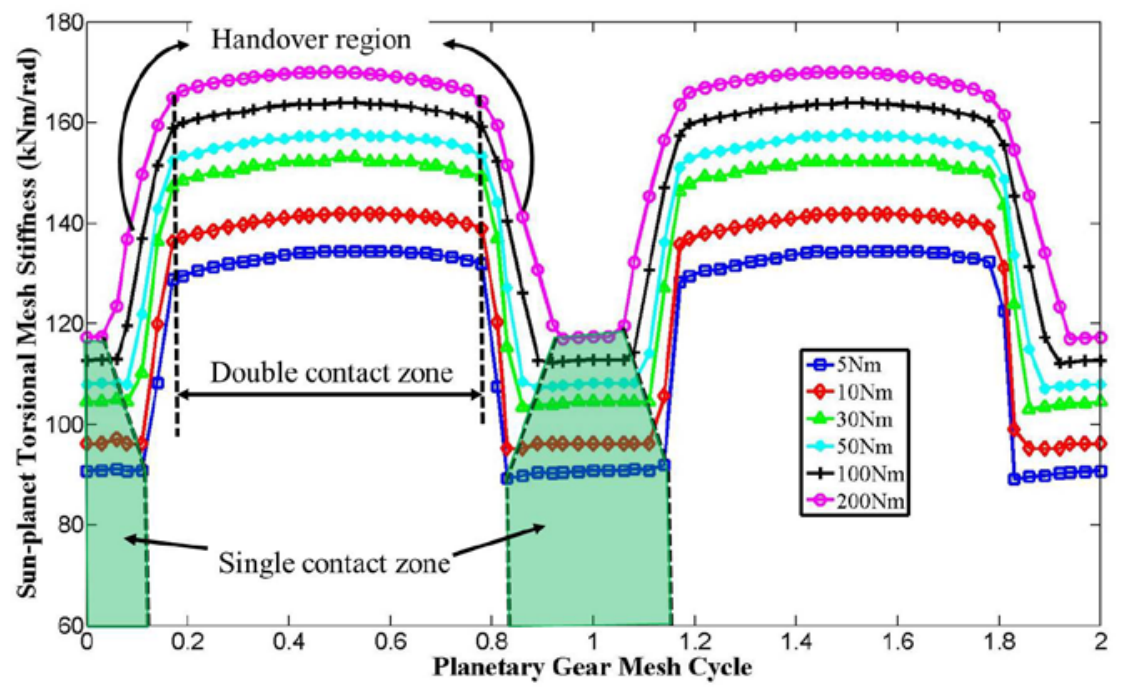

Fig. 2 Sun-planet subsystem torsional stiffness under different torque levels

The major components that make up the combined mesh stiffness result given in Fig.2 include (i) the gear body stiffness, (ii) the teeth bending stiffness and (iii) the nonlinear Hertzian contact stiffness [18], each of which has been shown to be a function of roll angle. During one gear mesh cycle, the mesh stiffness can be divided into three sections, that is, the double contact zone, single contact zone and a transition zone between the single and double zones, which can be defined as the handover region. The handover region is still in the double pair of teeth contact and it consists of an approach zone and a recess zone. From the results, it was found that as the input loads increased, the mesh stiffness varied slightly due to the Hertzian contact stiffness [22]. The relative difference between the torsional mesh stiffness under $5 \mathrm{Nm}$ load and the torsional mesh stiffness under $200 \mathrm{Nm}$ load was 23.5\%. Another important finding was that the length of the single contact zone would decrease with the increase of the input load while the length of the handover region (actually still the double contact zone) would be enlarged with the increase of the torque load.

\subsubsection{Isolated ring-planet (rp1) subsystem torsional stiffness}

The mesh stiffness variation of the ring-planet mesh was obtained from the isolated ring-planet pair model, which shares the same planet gear of the isolated sun-planet subsystem but with different meshing faces, as shown in Fig. 1(d). The outer rim of the ring gear was fully constrained, with no rotation and the planet was 
allowed the same movement as in the overall model, with the rigid carrier arm constraining the planet movement inside the ring gear. However, when the planet moves to each simulated position, its inner hub was again constrained so as not to move in the radial direction. To transmit the same input torque from the sun gear hub, a torque $T_{r p 1}=\left(Z_{p} / Z_{s}\right) \times T_{s p 1}$ was applied to the planet gear hub and $\Delta \theta_{r p 1}$ denotes the resulting elastic angular rotation of the planet gear hub. The torsional stiffness $K_{r p 1}$ was also obtained using equation (1). The corresponding ring-planet torsional stiffness results are shown in Fig. 3.

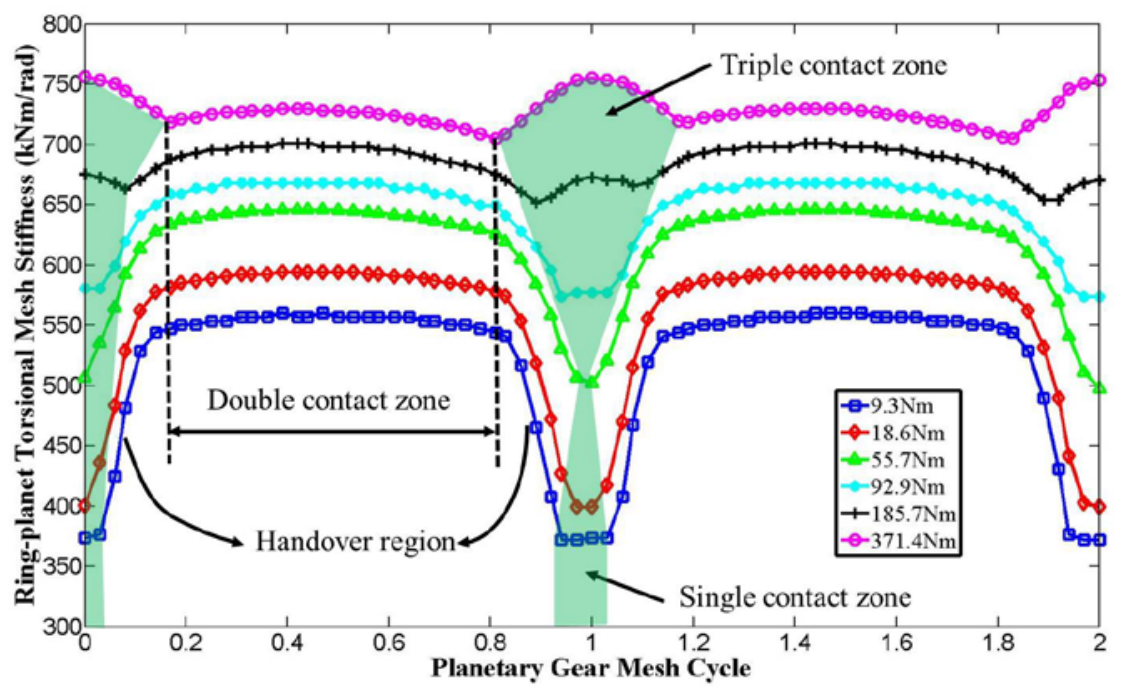

Fig. 3 Ring-planet torsional mesh stiffness under different torque levels

The ring-planet subsystem includes one external gear and one internal gear. Similar to the sun-planet mesh, the major components of the ring-planet mesh stiffness are: (i) the gear body stiffness, (ii) the teeth bending stiffness and (iii) the Hertzian contact stiffness. The relative phase between the ring-planet and sun-planet results for the shared planet can be calculated according to reference [21] and it is found to be 0.02 of the mesh cycle, which means the start mesh point of the ring-planet is also at the single contact zone, as shown in Fig. 3. The section during $0 \sim 0.03$ mesh cycle is the single contact zone and the section during $0.2 \sim 0.8$ mesh cycle is the double contact zone. The handover region can still be observed in the ring-planet mesh stiffness curve. However, with the increase of the load, it is interesting to note there is large variation in the single tooth zone mesh stiffness where this single tooth contact becomes triple teeth contact as the higher mesh forces are applied, as shown in Fig. 4. 

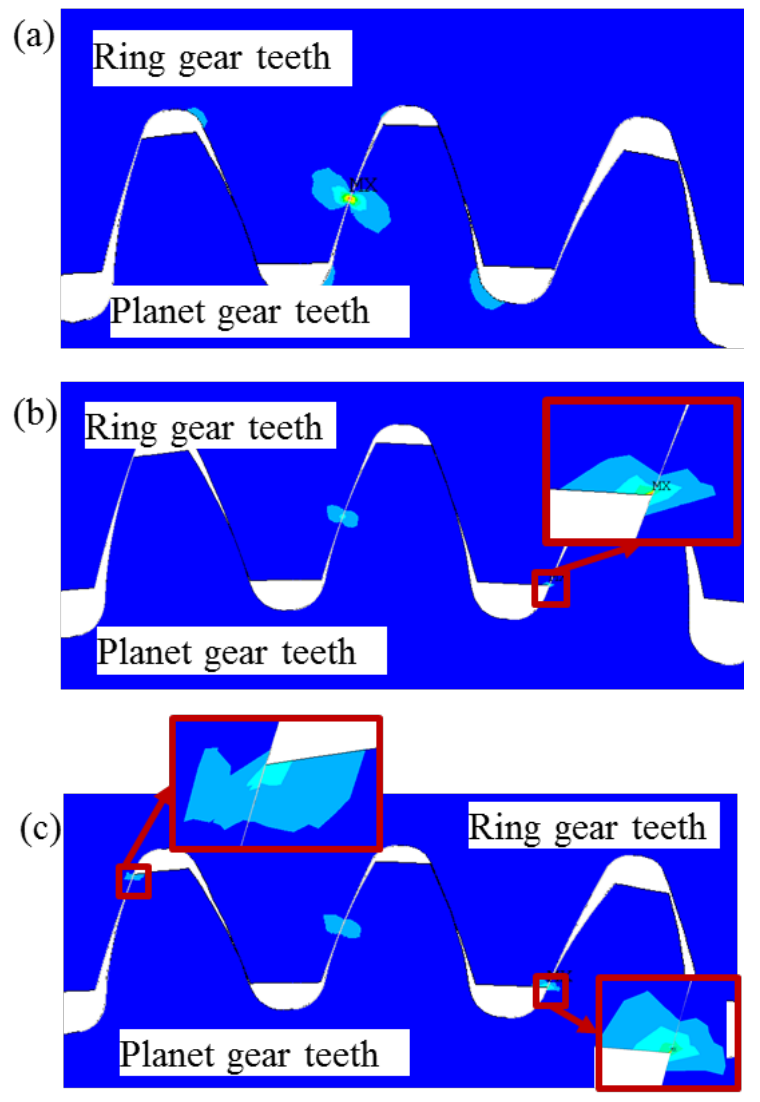

Fig. 4 Ring-planet Von Misses stress distribution under different loads, (a) 9.3Nm with single tooth contact, (b) $55.7 \mathrm{Nm}$ with double tooth contact, (c) $371.4 \mathrm{Nm}$ with triple tooth contact

\subsubsection{Influence of carrier arm stiffness}

As an important component, the carrier arm bears large load and the flexibility of the carrier arm can put great influence on load distribution and the resulting operational gearbox noise level. However, the structure of the carrier arm can be very complex and including all the details into the FEA software can cause great difficulty in the computation efficiency. Instead, the carrier arm was considered as a beam with a square cross-section, as shown in Fig. 1(b). A single branch was considered in this model and the combined sunplanet-ring mesh can be modelled either with a flexible carrier arm or with a rigid carrier arm. The outer rim of the ring gear was fully fixed and the planet gear was connected with the carrier arm with rigid bearing (MPC 184 revolute). The input torque load $T_{s p r 1}=T_{s p 1}$ was applied to the sun gear hub. If $\Delta \theta_{s p r 1}$ denotes the elastic angular rotation of the sun gear hub, the torsional stiffness $K_{\text {spr1 }}$ can be obtained using equation (1). The corresponding mesh stiffness curves under different loads are shown in Fig. 5. 


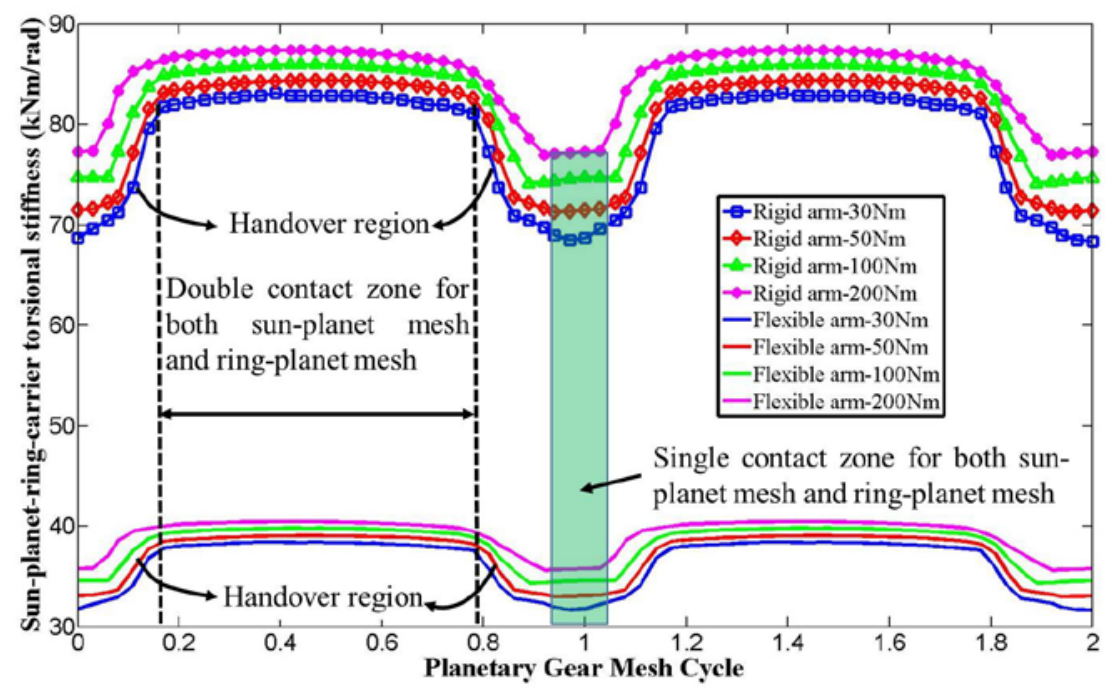

Fig. 5 Comparison of sun-planet-ring mesh stiffness with flexible carrier arm and with rigid carrier arm

As this stiffness was a combination of the sun-planet and ring-planet mesh stiffness, the section of $0.2 \sim 0.8$ mesh cycle is the double contact zone for both meshes and the section of $0 \sim 0.03$ mesh cycle is the single contact zone for both meshes. A combination of ring-planet single contact and sun-planet double contact zone can be found in the other sections because the contact ratio for the sun-planet is much smaller than that of the ring-planet. Therefore, the start meshing point of this model is within the single contact zone. Additionally, as shown in Fig. 5, the overall amplitude of the sun-planet-ring torsional mesh stiffness with the flexible arm is smaller than the overall amplitude of the sun-planet-ring mesh with the rigid arm. This is because the flexible carrier arm can add flexibility into the system and bring down the overall sun-planetring stiffness. However, it was found that the influence of the carrier arm flexibility was a constant factor in the sun-planet-ring overall stiffness and in fact, the value can be estimated from the beam bending theory. To achieve the same reaction force at the carrier arm hub, nominal torque $T_{c}=\left(1+Z_{r} / Z_{s}\right) T_{s}$ is applied to the carrier arm hub and $\Delta \theta_{c}$ is the angular rotation due to the torque $T_{c}$. According to the beam theory, $\Delta \theta_{c}=T_{c}$ l/3EI ( $l$ is the length of the beam and is equal to the sun-planet gear centre distance in this assumption. $E$ and I are the elastic modulus and second moment of area respectively). As a result, as long as the dimension of the beam cross section is chosen, the stiffness of the carrier arm can be estimated as [23],

$$
K_{c}=\frac{T_{c}}{\Delta \theta_{c}}=\frac{3 E I}{l}
$$




\subsubsection{Overall planetary gear torsional stiffness}

The overall planetary gear mesh stiffness variation can also be obtained from the planetary gear sets with the three planet model, as shown in Fig. 1(a). The outer rim of the ring gear was again constrained not to rotate and the torque $T_{s o}=3 T_{s p 1}$ was applied on the sun gear hub. If $\Delta \theta_{s o}$ denotes the resulting elastic angular rotation of the sun gear hub, the overall torsional stiffness $K_{s o}$ can be obtained using equation (1), as shown in Fig. 6 . The overall planetary gear mesh stiffness includes the sun-planet-ring mesh stiffness from the 3 planet branches and also the carrier arm stiffness.

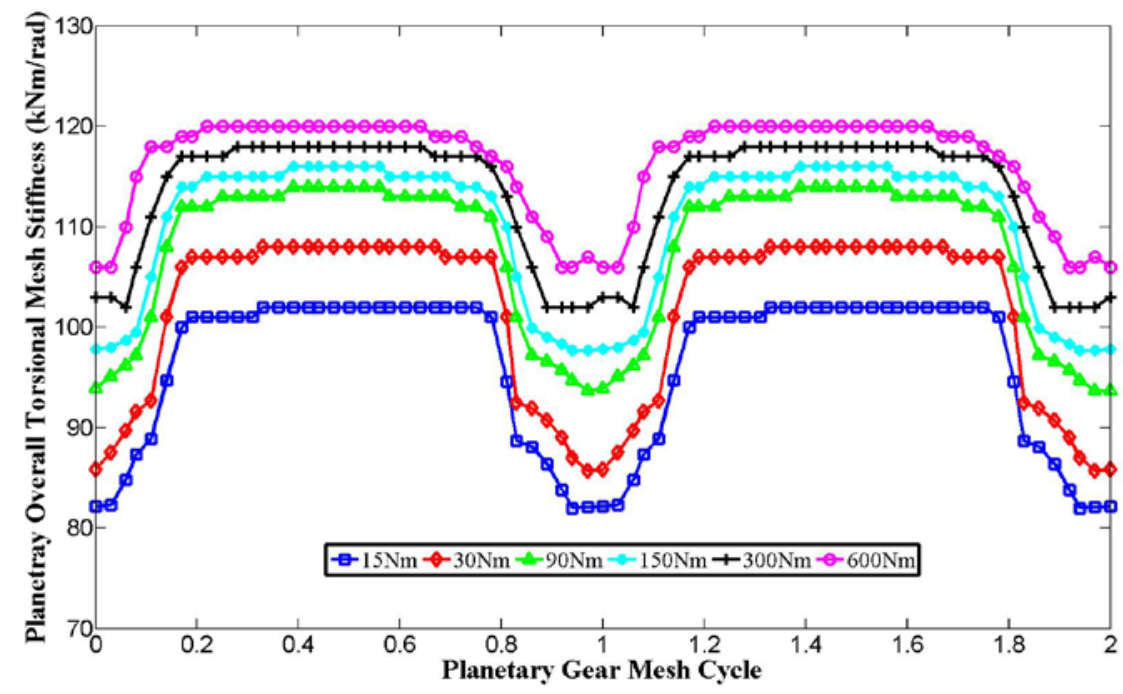

Fig. 6 Overall planetary gear torsional stiffness with 3 planets and flexible carrier arm

\section{Predictive Models for Planetary Gear Torsional Stiffness}

In a planetary gear set, there are several sun-planet gear pairs and several ring-planet gear pairs meshing simultaneously. While each of the sun-planet meshes has the same mesh stiffness shape variation, they are not necessarily in phase with each other. Similar situation exists for the ring-planet meshes. The stiffness of the $n$th sun-planet pair $\left(K_{s p n}\right)$ with respect to the $1^{\text {st }}$ sun-planet pair $\left(K_{s p 1}\right)$ and the stiffness of the nth ringplanet pair $\left(K_{r p n}\right)$ with respect to the $1^{\text {st }}$ ring-planet pair $\left(K_{r p 1}\right)$ are given as [21],

$$
\begin{aligned}
& K_{s p n}=K_{s p 1}\left(\theta-\gamma_{s n} \theta_{m}\right), \\
& K_{r p n}=K_{r p 1}\left(\theta-\gamma_{r n} \theta_{m}-\gamma_{r s} \theta_{m}\right),
\end{aligned}
$$

where $\theta_{m}$ is the rotation angle of the carrier arm in one mesh period and $\theta_{m}=2 \pi / Z_{r}$. The mesh phases $\gamma_{s n}, \gamma_{r n}$ and $\gamma_{r s}$ can be calculated according to reference [21]. For example, the gear meshes of the gear set used in this research were found to be $\gamma_{s n}=0$ and $\gamma_{r n}=0$. As a result, the relationships of $K_{s p n}=K_{s p 1}$ and $K_{r p n}=K_{r p 1}$ can 
be derived. However, for more general situations, the sun-planet mesh phases will not be identical to each other and similarly with the ring-planet mesh phases. If the planet gears are still kept equally distributed, the sun gear tooth number being 19 , the planet gear tooth number being 32 and the ring gear tooth number being 83, then the results of $\gamma_{s 2}=1 / 3$ and $\gamma_{r 2}=1 / 3, \gamma_{s 3}=2 / 3$ and $\gamma_{r 3}=2 / 3$ can be found. Therefore, there will be a phase shift between the different sun-planet-ring branches, but the peak-to-peak magnitudes will be the same. $\gamma_{r s}$ can also result in a phase shift between the sun-planet mesh and the ring-planet mesh at the same branch, though the magnitude of the stiffness value will not be affected. By this way, the mesh stiffness on different branches can be obtained based on the stiffness on the $1^{\text {st }}$ sun-planet-ring mesh, which has been calculated in section 2.2.

\subsection{Gear mesh stiffness determined from a pinion hub and a gear hub perspective}

As discussed in section 2.2, the isolated sun-planet torsional stiffness was calculated from the sun gear hub while the isolated ring-planet torsional stiffness was calculated from the planet gear hub. The carrier arm stiffness was also calculated from the carrier arm hub perspective. However, the overall planetary gear torsional stiffness was calculated from the sun gear hub. A discussion is needed to find the relationships between the torsional stiffnesses calculated from different hub perspectives. An external pair of gears was considered in Fig. 7, where the combined torsional mesh stiffness can be calculated from either the pinion hub or the gear hub. 


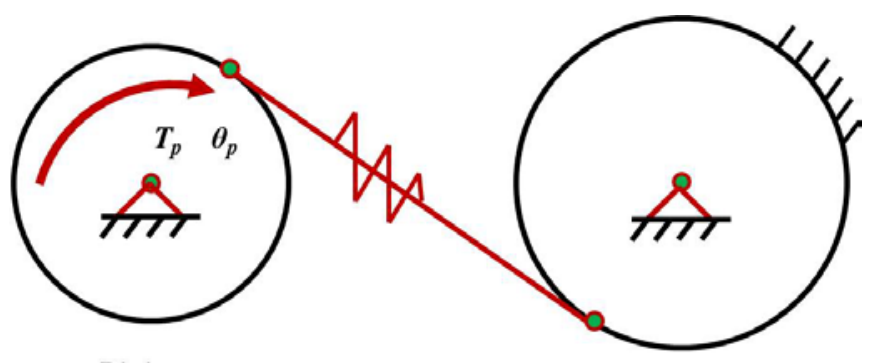

Pinion

Gear

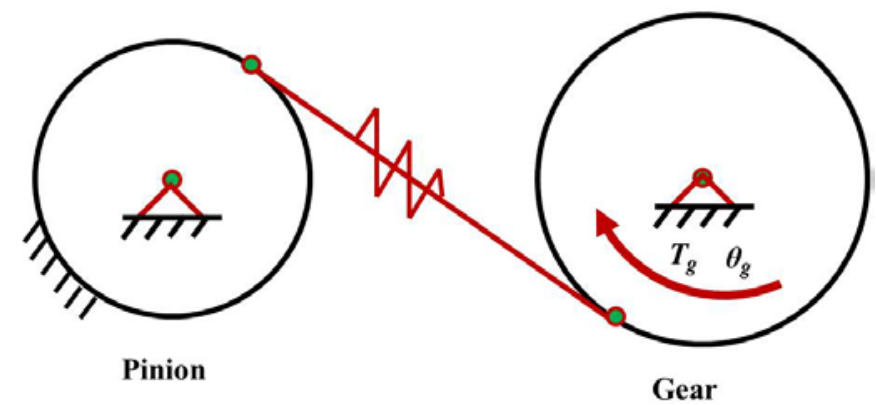

Fig. 7 Example of a gear pair

As shown in Fig. 7, when there is torque $T_{\text {pin }}$ applied on the pinion hub, the gear was constrained not to rotate. $\Delta \theta_{\text {pin }}$ is the total rotation angle measured from pinion hub at this condition. As a result, $K_{\text {pin }}=T_{\text {pin }} / \theta_{\text {pin }}$ is the combined torsional mesh stiffness calculated from the pinion hub. To achieve the same mesh force between the teeth, torque $T_{g}=\left(Z_{g} / Z_{p i n}\right) \cdot T_{\text {pin }}$ was applied on the gear hub and the pinion was constrained not to rotate. $\Delta \theta_{g}$ is the total rotation angle measured from pinion hub at this condition. As a result, $K_{g}=T_{g} / \theta_{g}$ is the combined torsional mesh stiffness calculated from the gear hub. The same mesh force in the two situations will result in the same mesh displacement, which can be expressed as $\theta_{\text {pin }} R_{\text {pin }}=\theta_{g} R_{g}$. Finally, the relationship of the combined mesh stiffness calculated from different hubs is,

$$
K_{\text {pin }}=\frac{T_{p i n}}{\theta_{\text {pin }}}=\frac{\left(\frac{Z_{p i n}}{Z_{g}}\right) \cdot T_{g}}{\left(\frac{R_{g}}{R_{p i n}}\right) \cdot \theta_{g}}=\frac{Z_{p i n} R_{p i n}}{Z_{g} R_{g}} K_{g}=u^{2} K_{g}
$$

where $u$ is the gear speed ratio. From equation (4), it was found that when the gear mesh stiffness value is calculated from the different hub, there is a gear speed ratio squared relationship between the two stiffnesses. This relationship indicates that the ring-planet gear stiffness calculated from the planet gear hub and carrier arm stiffness calculated from the carrier arm hub also have to be scaled with the corresponding speed ratio squared when considering them from the sun gear hub and they become as,

$$
K_{r p}^{s}=\left(\frac{Z_{s}}{Z_{p}}\right)^{2} K_{r p}=K_{r p} /\left(\frac{Z_{p}}{Z_{s}}\right)^{2}
$$




$$
K_{c}^{s}=\left(\frac{Z_{s}}{Z_{s}+Z_{r}}\right)^{2} K_{c}=K_{c} /\left(1+\frac{Z_{r}}{Z_{s}}\right)^{2}
$$

\subsection{Predictive models of the planetary gear torsional stiffness}

In the planetary gear system, the sun-planet mesh, ring-planet mesh and the carrier arm were found to be the fundamental components, whose stiffness values can be used to predict the overall planetary gear mesh stiffness. Initially, only the $1^{\text {st }}$ sun-planet-ring branch has been considered and this predictive model is equivalent to the FEA model shown in Fig. 1(b). A model having torsional springs connected in series can be used, as shown in Fig. 8.

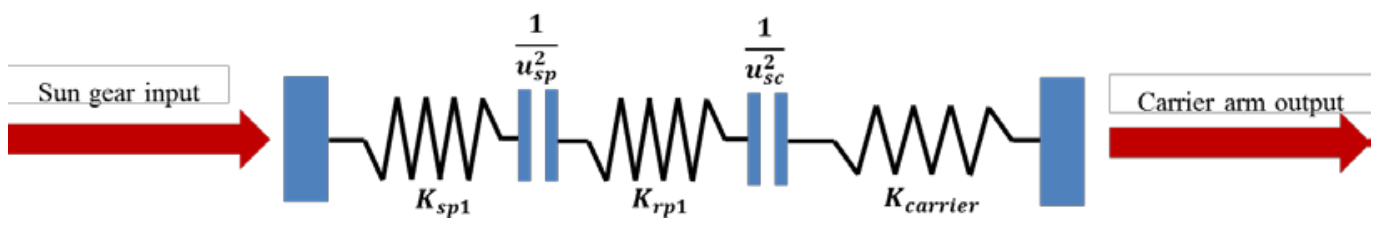

Fig. 8 Predictive model for a single branch of the sun-planet-ring mesh stiffness

As all the stiffness values are considered from the sun gear hub, the values of the planet-ring mesh and the carrier arm stiffness have to be scaled with the corresponding gear ratios. The single branch sun-planet-ring torsional stiffness can then be calculated as,

$$
K_{s p r 1}^{\prime}=\left(\frac{1}{K_{s p 1}}+\frac{1}{K_{r p 1}^{s}}+\frac{1}{K_{c}^{s}}\right)^{-1}=\left(\frac{1}{K_{s p 1}}+\frac{u_{s p}^{2}}{K_{r p 1}}+\frac{u_{s c}^{2}}{K_{c}}\right)^{-1},
$$

where $K_{\text {spr } 1}$ is the sun-planet-ring torsional mesh stiffness calculated using the prediction model. The ratio $u_{s p}=Z_{p} / Z_{s}$ is the sun-planet gear ratio and the ratio $u_{s c}=1+Z_{r} / Z_{s}$ is the sun-carrier speed ratio. The analytical results from equation (6) for input loads of $50 \mathrm{Nm}$ and $100 \mathrm{Nm}$ for the single planet branch case are shown in Fig. 9, along with the comparison of the FEA results modelled with the flexible carrier, originally shown in Fig. 5. 


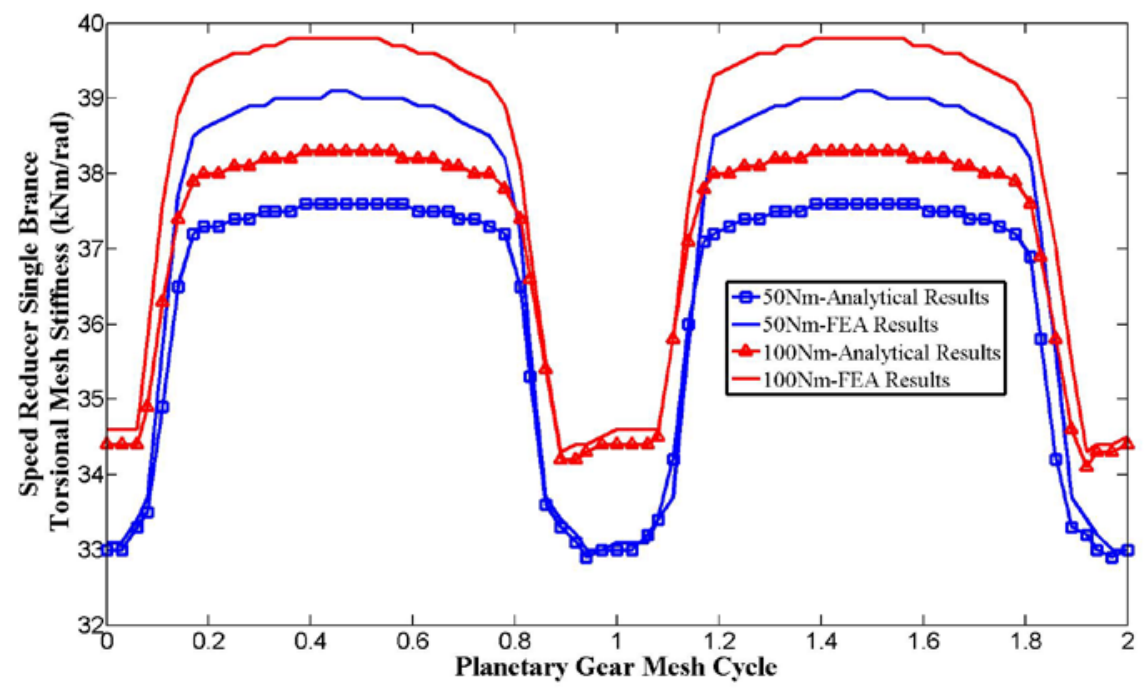

Fig. 9 Comparison between the single branch analytical and FEA torsional stiffness

The stiffness values at the starting point and at the 0.5 mesh cycle point are selected to compare the deviation between the analytical and FEA results. This is because the starting mesh point is in the zone where both meshes are in their own single contact zone and the mesh point at the 0.5 mesh cycle is in the zone where both meshes are in their own double contact zone. As shown in Fig. 9, the difference between the analytical result and the FEA result is minimal at the starting mesh point while the difference between the two at the 0.5 mesh cycle position is found to be largest during the whole mesh cycle. For example, the relative difference at the starting point under $100 \mathrm{Nm}$ is $0.6 \%$ while the relative difference at the 0.5 mesh cycle position is $3.8 \%$ with the same load. The discrepancies of the relative difference in the single and double zones are mainly caused by the calculation of the carrier arm stiffness, which was used in the predictive model, equation (6). This stiffness was calculated by equation (2) using the beam theory, however, the tooth will change from the single zone to double zone continuously with the gear rotation and this causes the loading condition of the carrier arm to subsequently change. Even though it was not so obvious, there will still be small discrepancies between the single zone and double zone for the value of the carrier arm.

The overall predictive model of torsional stiffness for the whole planetary system, which includes three branches of the sun-planet-ring mesh and the carrier arm, can also be established. In the predictive model, the torsional stiffness of every sun-planet-ring branch can be treated as torsional springs connected in series as shown in Fig. 10. 


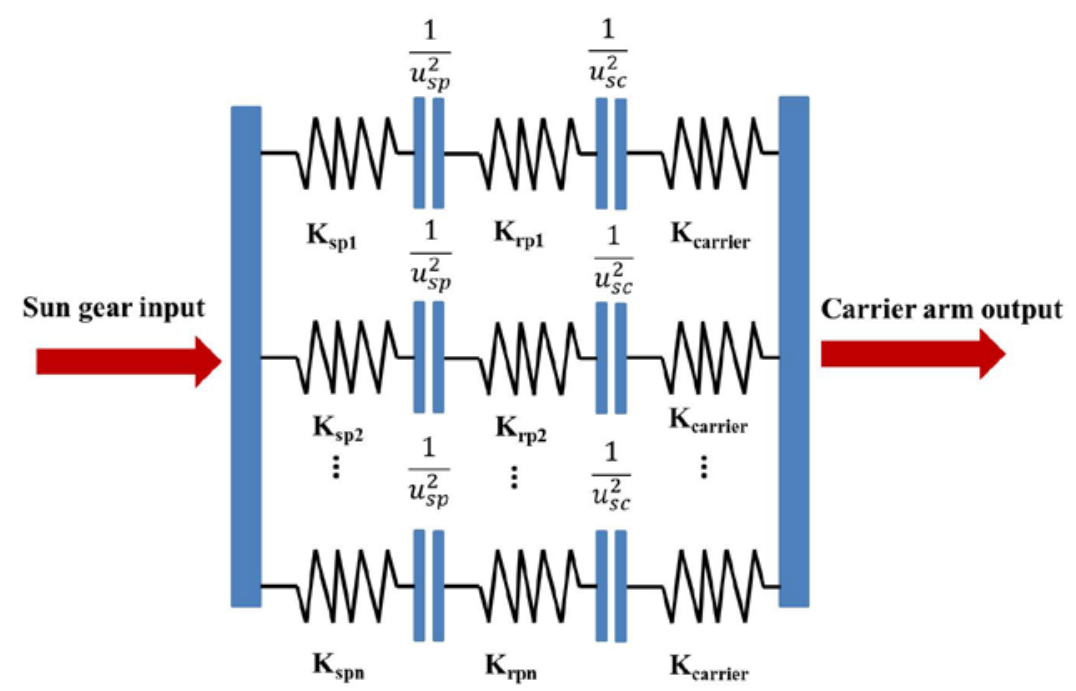

Fig. 10 Prediction model for the overall planetary gear

The stiffness of other planet branches $K_{\text {spr2 }}$ and $K_{\text {spr3 }}$ can be obtained using the phase relationship from equation (5) and then the overall planetary gear torsional stiffness can be calculated as,

$$
\begin{aligned}
& K_{s o}^{\prime}=K_{s p r 1}^{\prime}+K_{s p r 2}^{\prime}+K_{s p r 3}^{\prime} \\
& =\frac{1}{\frac{1}{K_{s p 1}(\theta)}+\frac{u_{s p}^{2}}{K_{r p 1}\left(\theta-\gamma_{r s} \theta_{m}\right)}+\frac{u_{s c}^{2}}{K_{c}}}+\frac{1}{\frac{1}{K_{s p 1}\left(\theta-\gamma_{s 2} \theta_{m}\right)}+\frac{u_{s p}^{2}}{K_{r p 1}\left(\theta-\gamma_{r 2} \theta_{m}-\gamma_{r s} \theta_{m}\right)}+\frac{u_{s c}^{2}}{K_{c}}} \\
& +\frac{1}{\frac{1}{K_{s p 1}\left(\theta-\gamma_{s 3} \theta_{m}\right)}+\frac{u_{s p}^{2}}{K_{r p 1}\left(\theta-\gamma_{r 3} \theta_{m}-\gamma_{r s} \theta_{m}\right)}+\frac{u_{s c}^{2}}{K_{c}}} .
\end{aligned}
$$

The analytical results for the overall planetary gear torsional stiffness are shown in Fig. 11, along with the FEA comparison. 


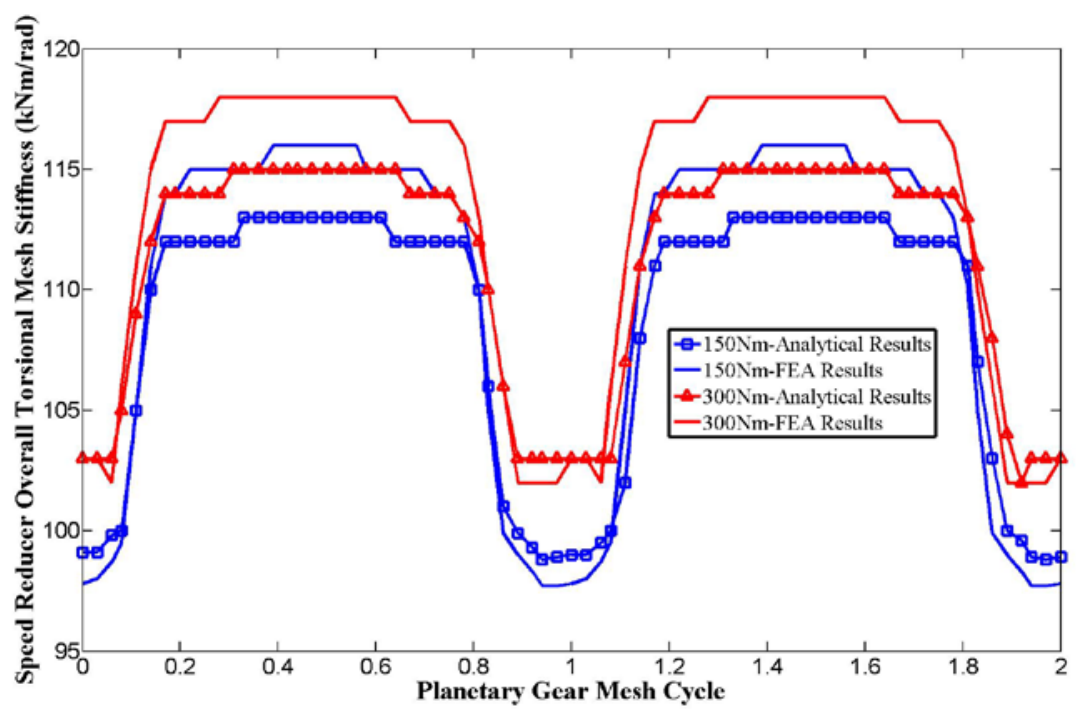

Fig. 11 Comparison between the overall planetary analytical and FEA torsional stiffness

Similarly with the single branch predictive model, a significant difference can be found at the 0.5 mesh cycle position, where the relative error is around 3.5\% under the load of $300 \mathrm{Nm}$. However, the most important finding is that the predictive model can capture the change of the overall mesh stiffness, no matter whether both meshes are in the single contact zone or in the double contact zone, or even in the combination zone.

For a planetary gear, there can be multiple movement arrangements when any of these members is selected as input or output and the predictive model shown in Fig. 10 is only for the case when sun gear is input and carrier arm is output. However, the advantage of this predictive model is that it can be modified to predict the overall torsional mesh stiffness for any gearbox train design because the individual mesh stiffness in the model is independent of the gear arrangement and only the gear speed ratio is needed to change correspondingly to fit for different arrangement. For example, when the carrier arm is selected as input and sun gear becomes output, the predictive model for this arrangement become as,

$$
K_{c o}^{\prime}=K_{r p s 1}^{\prime}+K_{r p s 2}^{\prime}+K_{r p s 3}^{\prime}
$$

where $K_{r p s n}^{\prime}(n=1,2,3)$ is the mesh stiffness of a single sun-planet-ring calculated from the carrier arm hub and it can be expressed as,

$$
K_{r p s n}^{\prime}=\left(\frac{1}{K_{c}}+\frac{u_{c s}^{2}}{K_{s p 1}\left(\theta-\gamma_{s n} \theta_{m}\right)}+\frac{u_{p c}^{2}}{K_{r p 1}\left(\theta-\gamma_{r n} \theta_{m}-\gamma_{r s} \theta_{m}\right)}\right)^{-1}
$$

where $u_{c s}=1 / u_{s c}=Z_{s} /\left(Z_{s}+Z_{r}\right)$ is the carrier-sun speed ratio and $u_{p c}=Z_{p} /\left(Z_{s}+Z_{r}\right)$ is the carrier-planet speed ratio. Equation (9) can also be expressed as, 


$$
K_{r p s n}^{\prime}=\left(\frac{1}{K_{c}}+\frac{1}{K_{s p 1}\left(\theta-\gamma_{s n} \theta_{m}\right) u_{s c}^{2}}+\frac{u_{s p}^{2}}{K_{r p 1}\left(\theta-\gamma_{r n} \theta_{m}-\gamma_{r s} \theta_{m}\right) u_{s c}^{2}}\right)^{-1}=K_{s p r n}^{\prime} u_{s c}^{2}
$$

From equation (10), it is found that $K_{r p s n}$ can also be estimated by multiplying $K_{\text {'sprn }}$ with the sun-carrier speed ratio and the stiffness results from the analytical equation (10) have been compared with the results from the FEA models, as shown in Fig. 12.

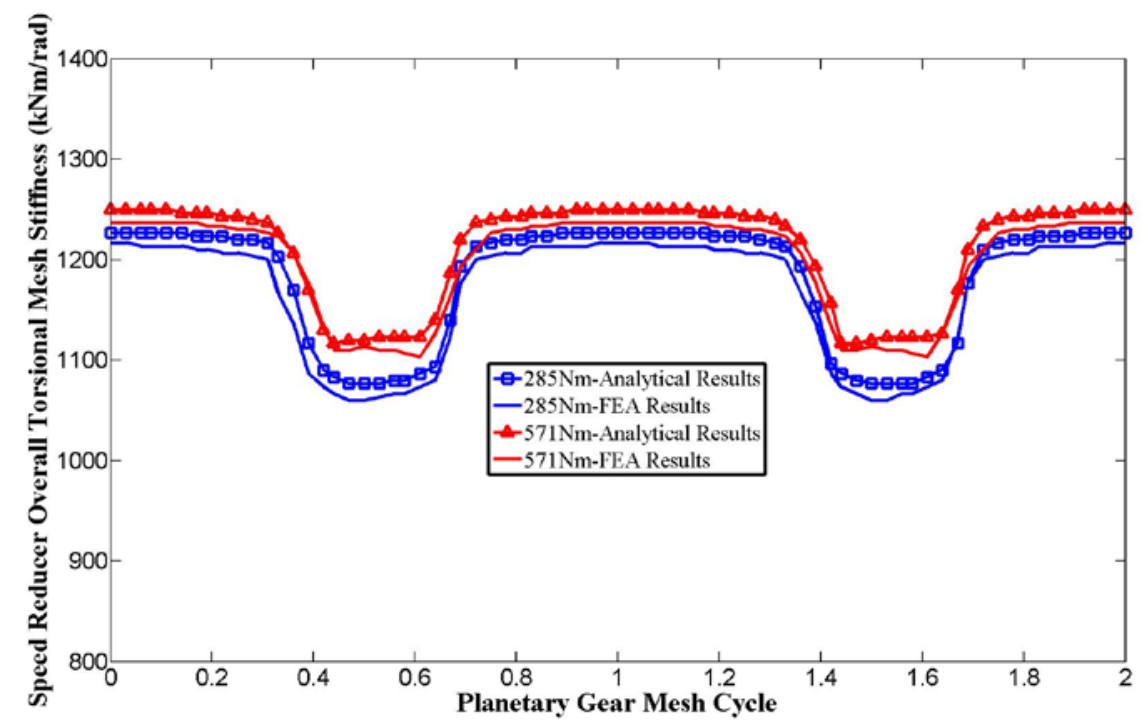

Fig. 12 Comparison between the single branch analytical and FEA torsional stiffness

As shown in Fig. 12, good agreement can be observed between the analytical result and FEA result and the relative error between the two results sets are within $4 \%$. Therefore, the relationships in equations (6-10) can be seen to be a powerful tool for estimating the overall mesh stiffness for any gear train design.

\section{Planetary gear crack sensitivity based on predictive models}

Cracks in gear teeth can occur due to various reasons, such as improper design and manufacturing, inadequate lubrication or transient overloading events. Once a crack occurs, the subsystem gear mesh stiffness will change [5-14] and consequently cause changes to the overall gear train stiffness and subsequently the dynamic system response. Vibration signal processing techniques tend to be used to detect these changes due to teeth damage [24]. It would be helpful to understand the influence of teeth crack damage on the overall planetary gear stiffness and the resulting crack sensitivity of different components.

A crack coefficient $\boldsymbol{C}_{\boldsymbol{j}}(\boldsymbol{j}=\boldsymbol{s p}, \boldsymbol{r} \boldsymbol{p})\left(0<\mathrm{C}_{\mathrm{j}} \leq 1\right)$ can be introduced to assist the analysis of the crack sensitivity on different components. The coefficient stands for the change ratio of the stiffness. For example, $\boldsymbol{C j}=\mathbf{0 . 1}$ means 
the corresponding stiffness will only have $10 \%$ left compared with the perfect mesh stiffness and could be considered a worst case. Correspondingly, $\boldsymbol{C} \mathbf{j}=\mathbf{1}$ means the resulting damage has caused no change of component stiffness.

If a crack occurs on the sun gear or in the planet gear on the sun gear side, the sun-planet mesh stiffness will be affected and the crack coefficient $\boldsymbol{C}_{\boldsymbol{s} p}$ can be assigned in the predictive model for a single branch of the sun-planet-ring mesh and equation (6) would then become,

$$
K_{s p r 1 \_c s p}^{\prime}=\left(\frac{1}{K_{s p 1} \cdot C_{s p}}+\frac{u_{s p}^{2}}{K_{r p 1}}+\frac{u_{s c}^{2}}{K_{c}}\right)^{-1} .
$$

While there is no damage on the other branches, the overall mesh stiffness would become,

$$
K_{s o_{-} c s p}^{\prime}=K_{s p r 1_{-} c s p}^{\prime}+K_{s p r 2}^{\prime}+K_{s p r 3}^{\prime} .
$$

The sun-planet crack sensitivity $\zeta_{s p}$ measured from the sun gear hub can then be calculated as,

$$
\zeta_{s p}=\frac{K_{s o}^{\prime}-K_{s o_{-} c p p}^{\prime}}{K_{s o}^{\prime}}=1-\frac{K_{s o_{-} c s p}^{\prime}}{K_{s o}^{\prime}} .
$$

If a crack occurs on the ring gear or in the planet gear on the ring gear side, the ring-planet mesh stiffness would be affected and the crack coefficient $\boldsymbol{C}_{\boldsymbol{r} p}$ can be assigned in the predictive model for a single branch of the sun-planet-ring mesh as a speed reducer and equation (6) would become,

$$
K_{s p r 1 \_c r p}^{\prime}=\left(\frac{1}{K_{s p 1}}+\frac{u_{s p}^{2}}{K_{r p 1} \cdot C_{r p}}+\frac{u_{s c}^{2}}{K_{c}}\right)^{-1} .
$$

While there is no damage on the other branches, the overall mesh stiffness would become,

$$
K_{s o_{-} c r p}^{\prime}=K_{s p r 1_{-} c r p}^{\prime}+K_{s p r 2}^{\prime}+K_{s p r 3}^{\prime} .
$$

The ring-planet crack sensitivity $\zeta_{\text {rp }}$ measured from the sun gear hub can be calculated as,

$$
\zeta_{r p}=\frac{K_{s o}^{\prime}-K_{s O_{\_} c r p}^{\prime}}{K_{s o}^{\prime}}=1-\frac{K_{s o_{-} c r p}^{\prime}}{K_{s o}^{\prime}} .
$$

\subsection{Crack sensitivity analysis}

If a crack is present in any of the gear components, there will be a range for the crack sensitivity depending on the overall stiffness. The minimum and maximum crack sensitivities for $\zeta_{\text {sp }}$ and $\zeta_{\mathrm{rp}}$ can be calculated as, 


$$
\begin{aligned}
& \zeta_{\mathrm{sp}}^{\min }=1-\frac{\mathrm{K}_{\mathrm{so} \_c s p}^{\prime}}{\min \left(\mathrm{K}_{\mathrm{so}}^{\prime}\right)} \text { and } \zeta_{\mathrm{sp}}^{\max }=1-\frac{\mathrm{K}_{\mathrm{so} \_c s p}^{\prime}}{\max \left(\mathrm{K}_{\mathrm{so}}^{\prime}\right)}, \\
& \zeta_{\mathrm{rp}}^{\min }=1-\frac{\mathrm{K}_{\mathrm{so} \_c r p}^{\prime}}{\min \left(\mathrm{K}_{\mathrm{so}}^{\prime}\right)} \text { and } \zeta_{\mathrm{rp}}^{\max }=1-\frac{\mathrm{K}_{\mathrm{so} \_c r p}^{\prime}}{\max \left(\mathrm{K}_{\mathrm{so}}^{\prime}\right)} .
\end{aligned}
$$

To find the minimum or the maximum value of the overall planetary stiffness, there are several things that should be noted. As discussed in section 2, the sun-planet and ring-planet meshes vary during the whole mesh cycle, which can be divided into the single contact zone, double contact zone and the handover region. With the increase of the input load, the stiffness value in these zones will vary slightly and the single contact zone can transfer to double, or even triple contact zone as shown in Fig. 3. Plus, the sun-planet and ring-planet meshes in the other branch would have a phase shift due to the existence of the mesh phase angle. As a result, there would be numerous stiffness combinations for the overall planetary stiffness and it would be a difficult task to assess the overall planetary stiffness. If a small input load, such as $15 \mathrm{Nm}$ was applied to the overall system, it can be assumed that the sun-planet and ring-planet stiffness values will achieve their maximum in the double contact zone and a minimum in the single contact zone. As shown in Fig. 2 and Fig. 3, the sun-planet mesh stiffnesses in the single and double contact zones are $89 \mathrm{kNm} / \mathrm{rad}$ and $134 \mathrm{kNm} / \mathrm{rad}$ respectively and the ring-planet mesh stiffnesses in the single and double contact zone are $370 \mathrm{kN} / \mathrm{rad}$ and $560 \mathrm{kNm} / \mathrm{rad}$ respectively. The carrier arm stiffness value is dependent on the shape of the beam cross-section and it was chosen as $2.16 \times 10^{3} \mathrm{kNm} / \mathrm{rad}$ initially. Each crack coefficient was changed from 0.1 to 1 and the corresponding crack sensitivity results are as shown in Fig. 13. 
(a)
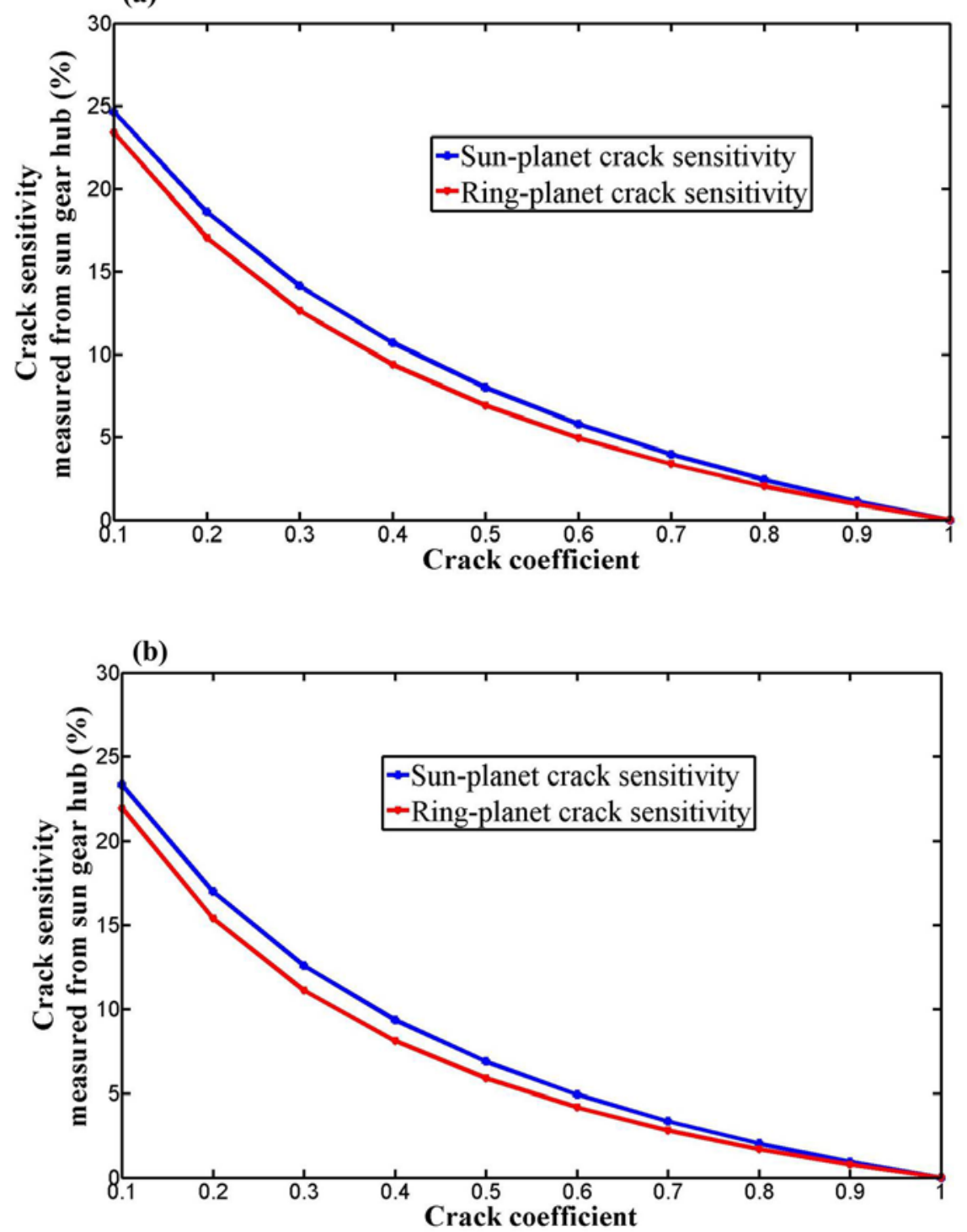

Fig. 13 Crack sensitivity measured from sun gear hub, (a) when all the meshes are in the single contact zone, (b) when all the meshes are in the double contact zone

Fig. 13 shows the resulting crack sensitivity results measured from the sun gear hub, where Fig. 13(a) provides the results when all the meshes are in the single contact zone and Fig. 13(b) provides the results when all the meshes are in the double contact zone. It was found that the cracks in the sun-planet mesh tended to be more sensitive than the cracks in the ring-planet mesh, regardless of whether all the meshes were in the single contact zone or in the double contact zone. Also, there was only slight difference between the crack sensitivities measured from the single contact zone and the crack sensitivities measured from the double contact zone. For example, when the crack coefficient $C_{s p}=0.1$, the sun-planet crack sensitivity in the single contact zone was $24.64 \%$ while the sun-planet crack sensitivity in the double contact zone was $23.34 \%$. As 
the maximum value will happen when all the sun-planet and ring-planet meshes are in their double contact zone and the minimum value will happen when all the meshes are in their single contact zone, the crack sensitivity in the other kinds of mesh phase combinations will vary between the results shown in Fig. 13(a) and Fig. 13(b).

\subsection{Influence of carrier arm stiffness on crack sensitivity}

In this study, the carrier arm stiffness can be calculated using beam bending theory, as shown in equation (2). If different beam dimensions were chosen, the carrier arm stiffness increases or decreases and therefore the overall planetary stiffness would also change correspondingly. As a result, the crack sensitivity will be affected by the choice of the carrier arm dimension. The carrier arm stiffness used in section 4.1 was $2.16 \times 10^{3}$ $\mathrm{kNm} / \mathrm{rad}$ and the power of 10 was assigned to this value to study the influence of carrier arm on crack sensitivity, as shown in Fig. 14. 
(a)

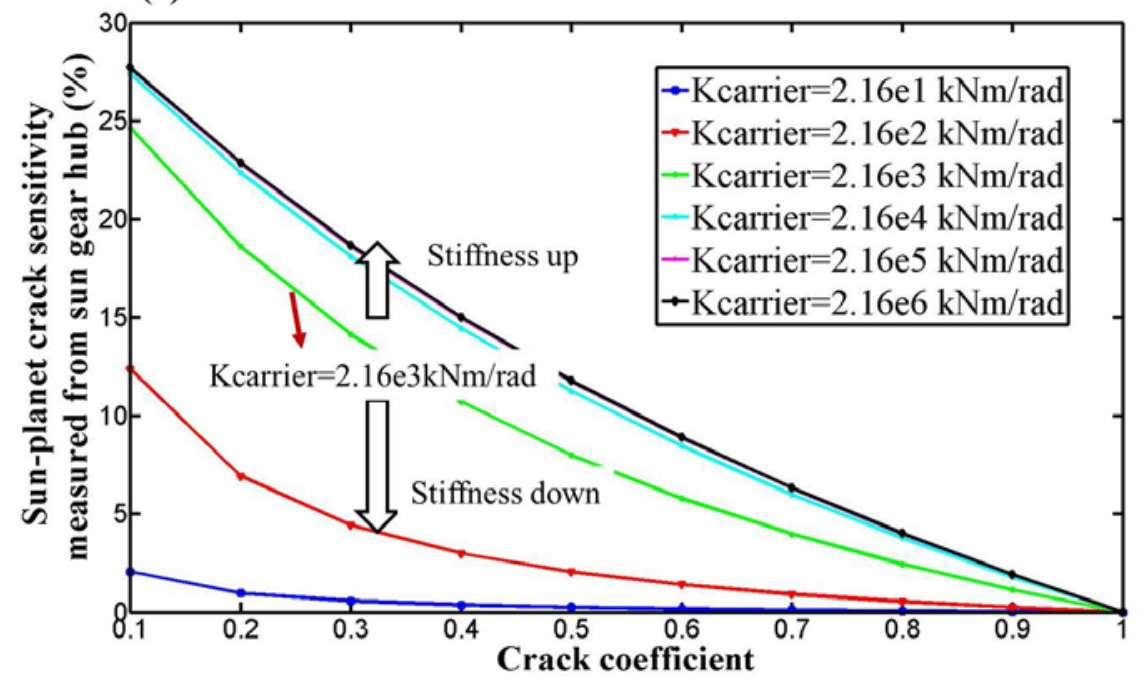

(b)

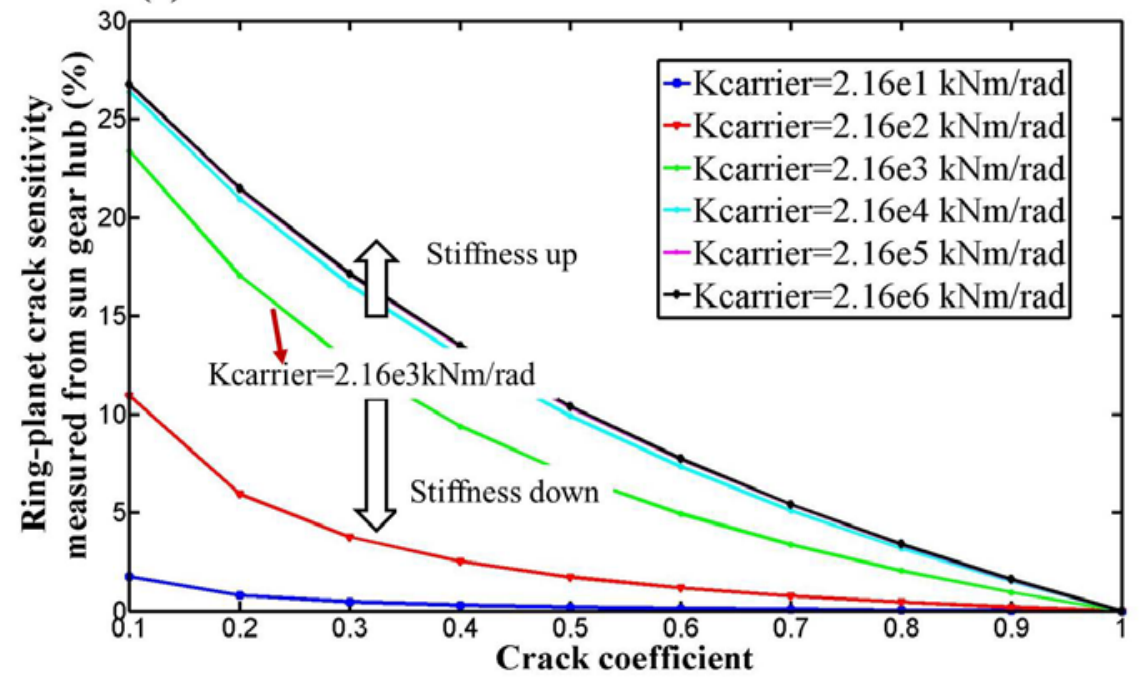

Fig. 14 Influence of carrier arm stiffness on crack sensitivity, (a) sun-planet crack sensitivity, (b) ring-planet crack sensitivity

Fig. 14 showed the influence of carrier arm stiffness on sun-planet and ring-planet crack sensitivities. It was found that both the sun-planet and ring-planet cracks became more sensitive when the carrier arm stiffness increased. Correspondingly, cracks would tend to be harder to be detected when the carrier arm stiffness becomes small. For example, when the crack coefficient $C_{s p}$ was assigned in the model, the sun-planet crack sensitivity was only $2 \%$ when the carrier arm stiffness was $2.16 \mathrm{e} 1 \mathrm{kNm} / \mathrm{rad}$ and the sun-planet crack sensitivity increases to $24.6 \%$ when the carrier arm stiffness increases to $2.16 \mathrm{e} 3 \mathrm{kNm} / \mathrm{rad}$. However, when the carrier arm stiffness increases to $2.16 \mathrm{e} 5 \mathrm{kNm} / \mathrm{rad}$, it was found it was difficult to enhance the sensitivity more. Similar results were found for the ring-planet crack sensitivity. 


\section{Discussion}

As the variations of the sun-planet and ring-planet mesh stiffnesses are the main internal vibration generation mechanisms for the gear system, the resultant mesh stiffness obtained in this paper can be used as input for planetary gear dynamic modelling to further improve the understanding of the gear system vibration response.

The subsystem mesh stiffness, like the sun-planet and ring-planet mesh stiffnesses, can be incorporated into the planetary dynamic models $[1,2]$ separately to study the dynamic response with or without gear cracks. In other words, there are two internal vibration generation sources in the planetary gear system. Different crack sizes and crack angles can be created to estimate their influence on the subsystem stiffness [25] and then these resultant stiffnesses can be used as input in the planetary gear system to obtain the time domain response or the frequency domain response.

The calculated sun-planet-ring mesh stiffness for one branch, as shown in Fig. 5 and Fig. 9, provided a way to combine all the subsystem stiffnesses together. By this way, the sun-planet and ring-planet stiffness behave as if they are in an in-series connection, as shown in Fig. 8, and they can be replaced with an equivalent stiffness, which has been widely used in planetary load sharing analytical models [26]. Another way of using this equivalent stiffness is to predict the sun gear radial orbit of a planetary gear set [27]. With the inclusion of the crack analysis in section 4 , the sun gear orbiting motion with cracks can be predicted to help further identification of the fault in the planetary system [28].

The predictive model for the overall planetary gear, as shown in Fig. 10, provided an easier way to estimate the overall mesh stiffness, which can also be extended to include multiple gearbox trains because it is independent of the gear arrangement and only the gear speed ratio is needed. One application of this method is that it can be used in wind turbine dynamic modelling to estimate the overall mesh stiffness of the gearbox stage. Usually, the gearbox modelling involves detailed gear dynamic equations [29, 30], which have also been introduced into the wind turbine modelling analysis [31, 32]. However, the wind turbine includes multiple components, like the rotor, shaft, generator and tower beside the gearbox. When the whole structure of the wind turbine was considered, the gearbox stage tends to be simplified as a constant stiffness [33] or even as rigid $[34,35]$. The predictive model developed in this paper can provide a reasonable estimation of the gearbox stage stiffness as long as the subsystem stiffness and the gear ratio are known. As a result, the 
variation of the gearbox stage can be considered in wind turbine dynamic modelling to help understand the overall wind turbine responses while avoiding the use of detailed gear dynamic equations.

\section{Conclusion}

A detailed calculation procedure for estimating the overall torsional mesh stiffness of a planetary gearbox has been developed in this paper. It included the FEA modelling of the individual sun-planet, ring-planet and carrier arm components and illustrated how they can be combined together via the gear speed ratios. The influence of the carrier arm stiffness has also been studied and showed that the effect of the torsional stiffness of the carrier arm was constant over the mesh cycle.

The predictive models for the overall torsional stiffness based on the subsystem models agreed well with the results from the FEA calculation and the relative error was found to be less than $4 \%$. It was found that the relationship between the overall model and subsystem models was directly related to the square of the speed ratios between the various components.

The crack sensitivity analysis based on the predictive models has been performed. The effect of a crack on the sun-planet mesh and ring-planet mesh stiffness has been introduced into the predictive models via a crack coefficient. It was found that the sensitivity measured from the sun gear hub was identical with those measured from the carrier arm. Also, it was found that the overall stiffness was most sensitive to the crack on the sun-planet mesh. Finally, the influence of the carrier arm stiffness on the crack sensitivity was discussed and it was found that both the sun-planet and ring-planet crack became more sensitive when the carrier arm became stiffer.

\section{References}

[1] A. Kahraman, Planetary gear train dynamics, J. Mech. Des. 116 (1994) 713-720.

[2] J. Lin, R.G. Parker, Analytical characterization of the unique properties of planetary gear free vibration, J. Vib. Acoust.121 (1999) 316-321.

[3] J. Lin, R.G. Parker, Planetary gear parametric instability caused by mesh stiffness variation, J. Sound Vib. 249 (2002) 129-145.

[4] A. Kahraman, Natural modes of planetary gear trains, J. Sound Vib. 173 (1994) 125-130. 
[5] Z. Chen, Y. Shao, Dynamic simulation of spur gear with tooth root crack propagating along tooth width and crack depth, Eng. Fail. Anal. 18 (2011) 2149-2164.

[6] Z. Chen, Y. Shao, Mesh stiffness calculation of a spur gear pair with tooth profile modification and tooth root crack, Mech. Mach. Theory 62 (2013) 63-74.

[7] R. Ma, Y. Chen, Research on the dynamic mechanism of the gear system with local crack and spalling failure, Eng. Fail. Anal. 26 (2012) 12-20.

[8] W. Yu, Y. Shao, C. K. Mechefske, The effects of spur gear tooth spatial crack propagation on gear mesh stiffness, Eng. Fail. Anal. 54 (2015) 103-119.

[9] A. Saxena, A. Parey, M. Chouksey, Effect of shaft misalignment and friction force on time varying mesh stiffness of spur gear pair, Eng. Fail. Anal. 49 (2015) 79-91.

[10] H. Ma, X. Pang, R. Feng, R. Song, B. Wen, Fault features analysis of cracked gear considering the effects of the extended tooth contact, Eng. Fail. Anal. 48 (2015) 105-120.

[11] O. Mohammed, M. Rantatalo, J. Aidanpaa, Improving mesh stiffness calculation of cracked gears for the purpose of vibration-based fault analysis, Eng. Fail. Anal. 34 (2013) 235-251.

[12] H. Ma, R. Song, X. Pang, B. Wen, Time-varying mesh stiffness calculation of cracked spur gears, Eng. Fail. Anal. 44 (2014) 179-194.

[13] Y. Pandya, A. Parey, Failure path based modified gear mesh stiffness for spur gear pair with tooth root crack, Eng. Fail. Anal. 27 (2013) 286-296.

[14] Y. Pandya, A. Parey, Simulation of crack propagation in spur gear tooth for different gear parameter and its influence on mesh stiffness, Eng. Fail. Anal. 30 (2013) 124-137.

[15] S. Li, Finite element analysis for contact strength and bending strength of pair of spur gears with machining errors, assembly errors and tooth modifications, Mech. Mach. Theory 42 (2007) 88-114.

[16] V.K. Ambarisha, R.G. Parker, Nonlinear dynamics of planetary gears using analytical and finite element models, J. Sound Vib.302 (2007) 577-595.

[17] J. Wang, I. Howard, Error analysis on finite element modelling of involute spur gears, J. Mech. Des. 128 (2006) 90-97. 
[18] J. Wang, I. Howard, The torsional stiffness of involute spur gears, P. I. Mech. Eng. C-J. Mech. 218(2004) 131-142.

[19] J. Wang, Numerical and experimental analysis of spur gears in mesh, PhD thesis, Curtin University of Technology, 2003.

[20] M. Inalpolat, A. Kahraman, A theoretical and experimental investigation of modulation sidebands of planetary gear sets, J. Sound Vibr., 323 (2009), 677-696.

[21] R.G. Parker, J. Lin, Mesh phasing relationships in planetary and epicyclic gears, J. Mech. Des. 126 (2004) 365-370.

[22] T. Keikbusch, D. Sappok, B. Sauer, I. Howard, Calculation of the combined torsional mesh stiffness of spur gears with two- and three-dimensional parametrical FE models, Strojniški vestnik - Journal of Mechanical Engineering 57(2011)11, 810-818.

[23] R.C. Hibberler, Engineering Mechanics- Statics, Prentice Hall, 2010.

[24] I. Howard, An investigation of vibration signal averaging of individual components in an epicyclic gearbox, Department of Defence, Aeronautical Research Laboratory, Propulsion Report 185, 1991.

[25] Z. Chen, Y. Shao, Dynamic simulation of planetary gear with tooth root crack in ring gear, Eng. Fail. Anal. 31 (2013) 8-18.

[26] A. Singh, Load sharing behaviour in epicyclic gears: Physical explanation and generalized formulation, Mech. Mach. Theory 45 (2010) 511-530.

[27] A. Banerjee, A model to predict sun gear radial orbit of a planetary gear set having manufacturing errors, Master thesis, The Ohio State University, 2012.

[28] S. Sheng, Y. Guo, An integrated approach using condition monitoring and modelling to investigate wind turbine gearbox design, National Renewable Energy Laboratory (NREL), Golden, Colorado, 2015.

[29] H. Ozguven, D. Houser, Mathematical models used in gear dynamics-a review, J. Sound Vibr., 121 (1988), 383-411.

[30] H. Ma, J. Zeng, R. Feng, X. Pang, Q. Wang, B. Wen, Review on dynamics of cracked gear systems, Eng. Fail. Anal. 55 (2015) 224-245. 
[31] M. Zhao, J.C. Ji, Nonlinear torsional vibrations of a wind turbine gearbox, Appl. Math. Modell., 39 (2015) 4928-4950.

[32] W. Shi, C. Kim, C. Chung, H. Park, Dynamic modelling and analysis of a wind turbine drivetrain using the torsional dynamic model, Int. J. Precis. Eng. Manuf., 14 (2013) 153-159.

[33] I.P. Girsang, J.S. Dhupia, E. Muljadi, M. Singh, L.Y. Pao, Gearbox and drivetrain models to study dynamic effects of modern wind turbines, IEEE Trans. Ind. Appl., 50 (2014) 3777-3786.

[34] J. Hall, D. Chen, Dynamic Optimization of drivetrain gear ratio to maximize wind turbine power generation- Part 1: system model and control framework, ASME J. Dyn. Sys., Meas., Control, 135 (2013) 011016.

[35] V. Dinh, B. Basu, Passive control of floating offshore wind turbine nacelle and spar vibrations by multiple tuned mass dampers, Struct. Control Health Monit., 22 (2015) 152-176.

\section{Appendix A Nomenclature}

$\boldsymbol{C}_{\boldsymbol{j}}$, the crack coefficient $(j=s p, r p)$;

$d_{i}$, pitch diameter $(i=s, p, r)$;

$E$, elastic modulus;

$I$, the second moment of area;

$K_{c}$, the stiffness of the carrier arm;

$K_{i}$, the gear mesh stiffness ( $i=p i n, g$ );

$K_{j n}, n$th torsional stiffness from the isolated subsystem $(j=s p, r p)$;

$K_{\text {sprn }}, n$th branch sun-planet-ring torsional stiffness using FEA;

$K_{\text {sprn, }} n$th branch sun-planet-ring torsional stiffness using the prediction model;

$K_{\text {so }}$, the overall planetary gear torsional stiffness measured from the sun gear hub using FEA;

$K_{\text {so }}$ the overall planetary gear torsional stiffness measured from the sun gear hub using the prediction model;

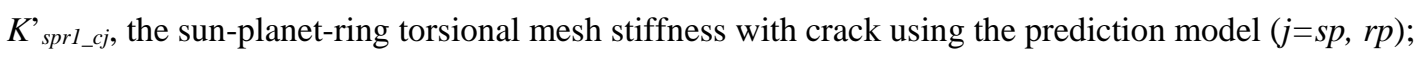

$K_{\text {so_cj, }}$, the overall planetary gear torsional stiffness with cracks measured from the sun gear hub using the prediction model $(j=s p, r p)$; 
$K^{\prime}{ }_{c o}$, the overall planetary gear torsional stiffness measured from the carrier arm hub using the prediction model;

$K_{r p}^{s}$, ring-planet mesh stiffness shifted to the sun gear hub;

$K_{c}^{s}$, carrier arm stiffness shifted to the sun gear hub;

$l$, the length of the beam;

$m_{n}$, gear module;

$\mathrm{N}$, the number of the planet gears;

$R_{i}$, the base radius ( $\left.i=p i n, g\right)$;

$T_{j 1}$, the input torque load on the gear hub for the subsystem $(j=s p, r p)$;

$T_{\text {spr } 1}$, the input torque load on the gear hub for one sun-planet-ring branch;

$T_{c}, T_{s}$, the input torque load on the carrier hub and sun gear hub;

$T_{\text {so }}$, the input torque load on the sun gear hub for the overall planetary gear;

$T_{i}$, the input torque load on the gear hub (i=pin, $g$ );

$\theta_{m}$, the rotation angle of the carrier arm in one mesh period;

$\Delta \theta_{i}$, the elastic angular rotation measured from the hub ( $\left.i=p i n, g\right)$;

$\Delta \theta_{c}$, the elastic carrier arm angular rotation;

$\Delta \theta_{j 1}$, the elastic angular rotation of the gear hub from the subsystem $(j=s p, r p)$;

$\Delta \theta_{\text {spr } 1}$, the elastic angular rotation of the gear hub from one branch;

$\Delta \theta_{s}$, the elastic angular rotation of the gear hub from the overall planetary gear;

$u_{s p}$, the sun-planet gear ratio;

$u_{s c}$, the sun-carrier speed ratio;

$u_{p c}$, the planet-carrier speed ratio;

$Z_{i}$, number of the gear teeth (i=s, $\left.p, r, p i n, g\right)$;

$\alpha_{i}$, pressure angle $(i=s, p, r)$;

$v$, Poisson's ratio;

$\zeta_{j}$, the crack sensitivity $(j=s p, r p)$;

$\gamma_{r n}$, mesh phasing between the various sun-planet meshes;

$\gamma_{s n}$, mesh phasing between the various ring-planet meshes; 